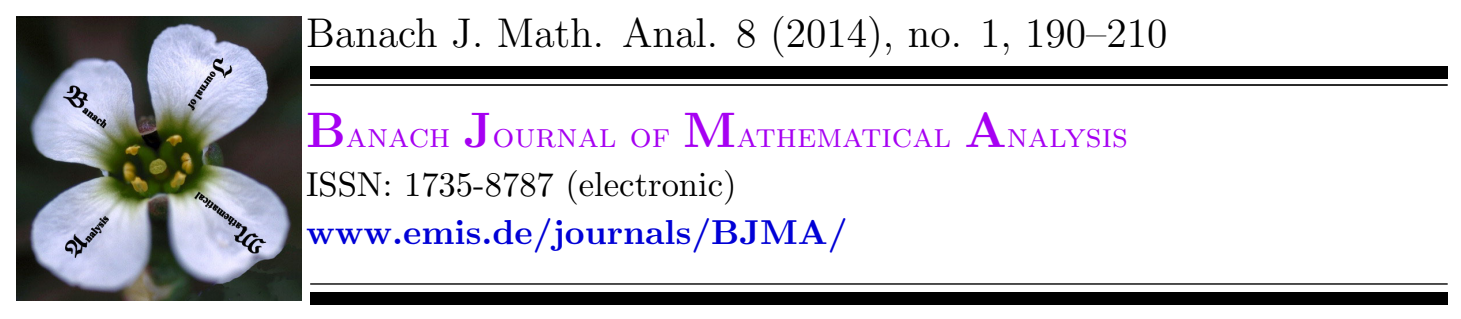

\title{
ABSOLUTELY SUMMING OPERATORS ON SEPARABLE LINDENSTRAUSS SPACES AS TREE SPACES AND THE BOUNDED APPROXIMATION PROPERTY
}

\author{
ÅSVALD LIMA ${ }^{1 *}$, VEGARD LIMA ${ }^{2}$, AND EVE OJA ${ }^{3}$ \\ Dedicated to the memory of Joram Lindenstrauss \\ Communicated by M. S. Moslehian
}

\begin{abstract}
Let $X$ be a Banach space and let $Y$ be a separable Lindenstrauss space. We describe the Banach space $\mathcal{P}(Y, X)$ of absolutely summing operators as a general $\ell_{1}$-tree space. We also characterize the bounded approximation property and its weak version for $X$ in terms of the space of integral operators $\mathcal{I}\left(X, Z^{*}\right)$ and the space of nuclear operators $\mathcal{N}\left(X, Z^{*}\right)$, respectively, where $Z$ is a Lindenstrauss space, whose dual $Z^{*}$ fails to have the Radon-Nikodým property.
\end{abstract}

\section{INTRODUCTION}

A Banach space is called a Lindenstrauss space (or an $L_{1}$-predual) if its dual space is isometrically isomorphic to an $L_{1}(\mu)$ space for some measure $\mu$. The class of Lindenstrauss spaces contains the $C(K)$ spaces and, more generally, the $M$-spaces, but it is a much wider class than the latter (see, e.g., [18], [20], [12], or [19, Part II, Chapter 4]).

The main aims of this paper are to describe absolutely summing operators on Lindenstrauss spaces and to demonstrate how any Lindenstrauss space whose

Date: Received: 15 May 2013; Revised: 11 June 2013; Accepted: 11 June 2013.

* Corresponding author.

2010 Mathematics Subject Classification. Primary: 47B10; Secondary: 46B20, 46B25, 46B28, 46E30, 47L05, 47L20.

Key words and phrases. Banach spaces, Banach operator ideals, bounded approximation properties, Lindenstrauss spaces. 
dual fails the Radon-Nikodým property can be used to characterize the classical bounded approximation property. This naturally leads us to study operators from and to the space $L_{1}[0,1]$.

In [13], we planted two-trunk trees in a Banach space $X$ and described the Banach space of absolutely summing operators $\mathcal{P}(C[0,1], X)$ from $C[0,1]$ to $X$ as an $\ell_{1}$-tree space on $X$ of two-trunk trees. In Section 2 of the present paper, we extend this description from $C[0,1]$ to an arbitrary separable Lindenstrauss space $Y$ : the space $\mathcal{P}(Y, X)$ will be described solely in terms of the space $X$ itself as a general $\ell_{1}$-tree space on $X$. In fact, every separable Lindenstrauss space gives rise to some kind of trees in an arbitrary Banach space $X$. In particular, the nice structure of classical Lindenstrauss spaces such as $C(\Delta)$, where $\Delta \subset[0,1]$ is the Cantor set, or $C[0,1]$ helps us to plant nice simple trees such as dyadic trees or two-trunk trees.

Recall that a Banach space $X$ is said to have the approximation property (AP) if there exists a net of finite rank operators $\left(S_{\alpha}\right) \subset \mathcal{F}(X, X)$ such that $S_{\alpha} \rightarrow$ $I_{X}$, the identity operator on $X$, uniformly on compact subsets of $X$. If $\left(S_{\alpha}\right)$ can be chosen with $\sup _{\alpha}\left\|S_{\alpha}\right\| \leq \lambda$ for some $\lambda \geq 1$, then $X$ has the $\lambda$-bounded approximation property ( $\lambda$-BAP). According to [16], we say that $X$ has the weak $\lambda$-bounded approximation property (weak $\lambda$-BAP) if for every Banach space $Y$ and every weakly compact operator $T \in \mathcal{W}(X, Y)$ there exists a net of finite rank operators $\left(S_{\alpha}\right) \subset \mathcal{F}(X, X)$ such that $S_{\alpha} \rightarrow I_{X}$ uniformly on compact sets in $X$ and $\lim \sup _{\alpha}\left\|T S_{\alpha}\right\| \leq \lambda\|T\|$.

In [15], we characterized the $\lambda$-BAP and the weak $\lambda$-BAP in terms of the space of integral operators $\mathcal{I}\left(X, C[0,1]^{*}\right)$ and the space of nuclear operators $\mathcal{N}\left(X, C[0,1]^{*}\right)$, respectively. In Section 3 , we show that $C[0,1]$ can be replaced by any Lindenstrauss space $Z$ such that $Z^{*}$ fails to have the Radon-Nikodým property and we still obtain characterizations of the $\lambda$-BAP and the weak $\lambda$ BAP. It is well known that $C[0,1]^{*}$ contains $L_{1}[0,1]$ as a subspace (in fact, as an $L$-summand), but $L_{1}[0,1]$ is not a dual space. Nevertheless, we prove that in the above-mentioned characterizations, $C[0,1]^{*}$ can be replaced by $L_{1}[0,1]$.

In Section 4, motivated by the main Theorem of Section 3 (Theorem 3.3) and applying results and ideas from Sections 2 and 3, we shall look at some structure of the spaces $\mathcal{I}\left(X, Z^{*}\right)$, where $Z$ is a Lindenstrauss space, and $\mathcal{I}\left(X, L_{1}[0,1]\right)$. In particular, we give reasonable formulas for computing respective integral norms of operators. We also show, e.g., that $\mathcal{I}\left(X, L_{1}[0,1]\right)$ is an L-summand in $\mathcal{I}\left(X, C[0,1]^{*}\right)$.

Our notation is standard. We consider Banach spaces over the real field $\mathbb{R}$. A Banach space $X$ will be regarded as a subspace of its bidual $X^{* *}$ under the canonical embedding $j_{X}: X \rightarrow X^{* *}$. We denote by $\mathcal{L}(X, Y)$ the Banach space of all bounded linear operators from $X$ to $Y$. Besides the operator ideal $\mathcal{P}$ of absolutely summing operators, we also need the ideals $\mathcal{I}$ and $\mathcal{N}$ of integral operators and of nuclear operators. Absolutely summing, integral, and nuclear norms of operators are denoted by $\|\cdot\|_{\mathcal{P}},\|\cdot\|_{\mathcal{I}}$, and $\|\cdot\|_{\mathcal{N}}$, respectively. For $\mathcal{P}$, $\mathcal{I}$, and $\mathcal{N}$, we refer to the books by Diestel, Jarchow, and Tonge [5], Pietsch [27], and Ryan [28]. 


\section{Absolutely summing operators on a SePARABle Lindenstrauss} SPACE AS A TREE SPACE

Although separable Lindenstrauss spaces seem not to have a transparent functional representation, they admit a useful description which is due to Lazar and Lindenstrauss [11] and Michael and Pełczyński [21] (see [12] or, e.g., [19, p. 165]).

Theorem 2.1 (Lazar, Lindenstrauss, Michael, Pełczyński). Let $Y$ be a separable Banach space. The following statements are equivalent.

(a) $Y$ is a Lindenstrauss space.

(b) $Y=\overline{\cup_{n=1}^{\infty} E_{n}}$ with $E_{n} \subset E_{n+1}$ and $E_{n}$ isometrically isomorphic to $\ell_{\infty}^{n}$ for every $n$.

(c) $Y=\overline{\cup_{n=0}^{\infty} F_{n}}$ with $F_{n} \subset F_{n+1}$ and $F_{n}$ isometrically isomorphic to $\ell_{\infty}^{m_{n}}$ for every $n$ and some $m_{0}<m_{1}<m_{2}<\cdots<m_{n}<m_{n+1}<\cdots$.

There are important separable Lindenstrauss spaces $Y$ which can be represented as in (c) in such a way that the spaces $F_{n}$ have simple useful bases $\left(y_{k, n}\right)_{k=1}^{m_{n}}$ and the system $\left(\left(y_{k, n}\right)_{k=1}^{m_{n}}\right)_{n=0}^{\infty}$ has a nice tree-like structure. (In fact, as we shall see below, any separable Lindenstrauss space gives rise to some tree-like structure.)

Example 2.2. Denote by $\ell_{\infty}[0,1]$ the Banach space of bounded functions on $[0,1]$. Consider the system $\left(\left(y_{k, n}\right)_{k=1}^{2^{n}}\right)_{n=0}^{\infty}$ in $\ell_{\infty}[0,1]$, where $y_{1,0}=\chi_{[0,1)}, y_{1,1}=$ $\chi_{[0,1 / 2)}, y_{2,1}=\chi_{[1 / 2,1)}, y_{1,2}=\chi_{[0,1 / 4)}, y_{2,2}=\chi_{[1 / 4,1 / 2)}, y_{3,2}=\chi_{[1 / 2,3 / 4)}, y_{4,2}=$ $\chi_{[3 / 4,1)}$, and so on, i.e., $y_{k, n}=\chi_{\left[\frac{k-1}{2^{n}}, \frac{k}{2^{n}}\right)}$ for $n=0,1, \ldots$ and $k=1, \ldots, 2^{n}$. Then $\left(\left(y_{k, n}\right)_{k=1}^{2^{n}}\right)_{n=0}^{\infty}$ is a dyadic tree in $\ell_{\infty}[0,1]$, since

$$
y_{k, n}=y_{2 k-1, n+1}+y_{2 k, n+1}
$$

for all $n=0,1, \ldots$ and $k=1, \ldots, 2^{n}$.

Denote $F_{n}=\operatorname{span}\left\{y_{k, n}: k=1, \ldots, 2^{n}\right\}$ and $M=\overline{\cup_{n=0}^{\infty} F_{n}} \subset \ell_{\infty}[0,1]$. Since $\sum_{k=1}^{2^{n}} y_{k, n}=\chi_{[0,1)}$ and $\left\|y_{k, n}\right\|=1$, it easily follows that

$$
\left\|\sum_{k=1}^{2^{n}} \lambda_{k} y_{k, n}\right\|=\max _{1 \leq k \leq 2^{n}}\left|\lambda_{k}\right|
$$

for all scalars $\left(\lambda_{k}\right)_{k=1}^{2^{n}}$.

Note that we can also consider $M \subset L_{\infty}[0,1]$.

Example 2.3. Let $Y=C(\Delta)$. Let $y_{1,0}=\chi_{\Delta}, y_{1,1}=\chi_{\Delta \cap[0,1 / 3]}, y_{2,1}=\chi_{\Delta \cap[2 / 3,1]}$, $y_{1,2}=\chi_{\Delta \cap[0,1 / 9]}, y_{2,2}=\chi_{\Delta \cap[2 / 9,1 / 3]}, y_{3,2}=\chi_{\Delta \cap[2 / 3,7 / 9]}, y_{4,2}=\chi_{\Delta \cap[8 / 9,1]}$, and so on. Then $\left(\left(y_{k, n}\right)_{k=1}^{2^{n}}\right)_{n=0}^{\infty}$ is a dyadic tree in $C(\Delta)$, since we have

$$
y_{k, n}=y_{2 k-1, n+1}+y_{2 k, n+1} \text {. }
$$

Denoting $F_{n}=\operatorname{span}\left\{y_{k, n}: k=1, \ldots, 2^{n}\right\}$, we have $C(\Delta)=\overline{\cup_{n=0}^{\infty} F_{n}}$. Since $\sum_{k=1}^{2^{n}} y_{k, n}=\chi_{\Delta}$ and $\left\|y_{k, n}\right\|=1$, it easily follows that

$$
\left\|\sum_{k=1}^{2^{n}} \lambda_{k} y_{k, n}\right\|=\max _{1 \leq k \leq 2^{n}}\left|\lambda_{k}\right|
$$

for all scalars $\left(\lambda_{k}\right)_{k=1}^{2^{n}}$. 
Example 2.4. Let $Y=C[0,1]$. Let $F_{n}$ denote the space of all linear splines on $[0,1]$ with knots $\left\{k / 2^{n}: k=0,1, \ldots, 2^{n}\right\}$. As in [13, Example 2.2], let $\left(g_{k, 2^{n}}\right)_{k=0}^{2^{n}}$ be the basis for $F_{n}$ defined by the conditions

$$
g_{k, 2^{n}}\left(\frac{k}{2^{n}}\right)=1 \quad \text { and } \quad g_{k, 2^{n}}\left(\frac{j}{2^{n}}\right)=0 \text { if } j \neq k,
$$

i.e., $g_{k, 2^{n}}$ are linear B-splines. Denote $y_{k, n}=g_{k-1,2^{n}}, n=0,1, \ldots$ and $k=$ $1, \ldots, 2^{n}+1$. Then $\left(\left(y_{k, n}\right)_{k=1}^{2^{n}+1}\right)_{n=0}^{\infty}$ is a two-trunk tree in $C[0,1]$ (for a definition of a two-trunk tree in a Banach space, see [13] or Remark 2.10 below). We also have $C[0,1]=\overline{\cup_{n=0}^{\infty} F_{n}}, \sum_{k=1}^{2^{n}+1} y_{k, n}=\chi_{[0,1]},\left\|y_{k, n}\right\|=1$, and

$$
\left\|\sum_{k=1}^{2^{n}+1} \lambda_{k} y_{k, n}\right\|=\max _{1 \leq k \leq 2^{n}+1}\left|\lambda_{k}\right|
$$

for all scalars $\left(\lambda_{k}\right)_{k=1}^{2^{n}+1}$.

Example 2.5. Let $Y$ be any separable Lindenstrauss space. Reformulating its representation (b) of Theorem 2.1, there exist subspaces $F_{n} \subset F_{n+1}$ with $F_{n}$ isometrically isomorphic to $\ell_{\infty}^{n+1}$ for every $n=0,1, \ldots$ By [21] or [12, p. 179] (see, e.g., [19, p. 166]) there exist bases $\left(y_{k, n}\right)_{k=1}^{n+1}$ in $F_{n}$ and a triangular matrix $A=\left(\left(a_{k, n}\right)_{k=1}^{n+1}\right)_{n=0}^{\infty}$ with $\sum_{k=1}^{n+1}\left|a_{k, n}\right| \leq 1, n=0,1, \ldots$ such that

$$
y_{k, n}=y_{k, n+1}+a_{k, n} y_{n+2, n+1}
$$

for all $n=0,1, \ldots$ and $k=1, \ldots, n+1$. Moreover

$$
\left\|\sum_{k=1}^{n+1} \lambda_{k} y_{k, n}\right\|=\max _{1 \leq k \leq n+1}\left|\lambda_{k}\right|
$$

for all scalars $\left(\lambda_{k}\right)_{k=1}^{n+1}$. Such a matrix $A$ was associated to $Y$ in [12] and was called a representing matrix of $Y$. The representing matrix is not uniquely determined. For a study of representing matrices and their connections with underlying separable Lindenstrauss spaces, the reader is referred to [12] (see also [19, pp. 165-169]).

Concerning Examples 2.2 and 2.3 above, let us point out the following connection.

Proposition 2.6. There exists an isometric isomorphism between the spaces $M$ and $C(\Delta)$.

Proof. Denote by $\left(\left(\bar{y}_{k, n}\right)_{k=1}^{2^{n}}\right)_{n=0}^{\infty}$ the dyadic tree in $M$ defined in Example 2.2. And let $\left(\left(y_{k, n}\right)_{k=1}^{2^{n}}\right)_{n=0}^{\infty}$ be the dyadic tree in $C(\Delta)$ defined in Example 2.3.

We shall denote $F_{n}=\operatorname{span}\left\{y_{k, n}: k=1, \ldots, 2^{n}\right\} \subset C(\Delta)$ and $G_{n}=\operatorname{span}\left\{\bar{y}_{k, n}\right.$ : $\left.k=1, \ldots, 2^{n}\right\} \subset M$. For $n=0,1, \ldots$, let $\theta_{n}: G_{n} \rightarrow F_{n}$ be the linear isometry which carries $\bar{y}_{k, n}$ to $y_{k, n}, k=1, \ldots, 2^{n}$. Then $\left.\theta_{n+1}\right|_{G_{n}}=\theta_{n}$ because

$$
\theta_{n+1}\left(\bar{y}_{k, n}\right)=\theta_{n+1}\left(\bar{y}_{2 k-1, n+1}+\bar{y}_{2 k, n+1}\right)=y_{2 k-1, n+1}+y_{2 k, n+1}=y_{k, n}, k=1, \ldots, 2^{n} \text {. }
$$

It follows that $\left.\theta_{m}\right|_{G_{n}}=\theta_{n}$ whenever $m \geq n$. 
We can now define $\theta: \cup_{n=0}^{\infty} G_{n} \rightarrow \cup_{n=0}^{\infty} F_{n}$ by $\theta x=\theta_{n} x$ whenever $x \in G_{n}$ for some $n$. The mapping $\theta$ is well-defined and linear. Clearly $\theta$ is an isometry. The desired isometric isomorphism will be the extension by continuity of $\theta$.

Let $Y$ be a separable Lindenstrauss space with a general structure as in Theorem 2.1 (c) above. Since $F_{n}$ is isometric to $\ell_{\infty}^{m_{n}}$, looking at the isometric copy of the unit vector basis, we see that there exists a basis $\left(y_{k, n}\right)_{k=1}^{m_{n}}$ in $F_{n}$ such that

$$
\left\|\sum_{k=1}^{m_{n}} \lambda_{k} y_{k, n}\right\|=\max _{1 \leq k \leq m_{n}}\left|\lambda_{k}\right|
$$

for all scalars $\left(\lambda_{k}\right)_{k=1}^{m_{n}}$. Extending [12, p. 179] (or [19, p. 165]), we call such a basis of $F_{n}$ admissible. If $\left(y_{k, n}\right)_{k=1}^{m_{n}}$ is an admissible basis in $F_{n}$, then its coordinate functionals in $F_{n}^{*}$ are of norm one. Hence there exist $y_{k, n}^{*} \in B_{Y^{*}}, k=1, \ldots, m_{n}$, such that $\left(\left(y_{k, n}\right)_{k=1}^{m_{n}},\left(y_{k, n}^{*}\right)_{k=1}^{m_{n}}\right)$ is a biorthogonal system.

We can now describe absolutely summing operators on separable Lindenstrauss spaces and calculate their norms (see Theorems 2.7 and 2.11 below). Recall that a linear operator $T: Y \rightarrow X$ is said to be absolutely summing if there exists a constant $C \geq 0$ such that

$$
\sum_{k=1}^{n}\left\|T y_{k}\right\| \leq C \sup \left\{\sum_{k=1}^{n}\left|y^{*}\left(y_{k}\right)\right|: y^{*} \in Y^{*},\left\|y^{*}\right\| \leq 1\right\}
$$

for every choice of elements $y_{1}, \ldots, y_{n}$ in $Y$. The minimum value of the constant $C$ is called the absolutely summing norm of $T$ and is denoted by $\|T\|_{\mathcal{P}}$.

Theorem 2.7. Let $X$ be a Banach space. Let $Y=\overline{\cup_{n=0}^{\infty} F_{n}}$ be a separable Lindenstrauss space with a structure as in Theorem 2.1 (c). Let $\left(y_{k, n}\right)_{k=1}^{m_{n}}$ be an admissible basis in $F_{n}$ and let $\left(y_{k, n}^{*}\right)_{k=1}^{m_{n}} \subset B_{Y^{*}}$ be functionals forming a biorthogonal system together with $\left(y_{k, n}\right)_{k=1}^{m_{n}} \subset Y$. If $T \in \mathcal{P}(Y, X)$, then

$$
T y=\lim _{n} \sum_{k=1}^{m_{n}} y_{k, n}^{*}(y) T y_{k, n}
$$

for all $y \in Y$ and

$$
\|T\|_{\mathcal{P}}=\sup _{n} \sum_{k=1}^{m_{n}}\left\|T y_{k, n}\right\|=\lim _{n} \sum_{k=1}^{m_{n}}\left\|T y_{k, n}\right\| .
$$

The proofs of Theorem 2.7 and Theorem 2.11 below will develop ideas from our paper [13, proof of Theorem 3.2] and they will use the following (folkloric) lemma (see [13, Lemma 3.1]).

Lemma 2.8. Let $X$ and $Y$ be Banach spaces, and let $T_{n} \in \mathcal{P}(Y, X)$. If the sequence $\left(T_{n}\right)$ is bounded in $\mathcal{P}(Y, X)$ and for every $y \in Y$ the limit $T y:=\lim _{n} T_{n} y$ exists, then $T \in \mathcal{P}(Y, X)$ and $\|T\|_{\mathcal{P}} \leq \sup _{n}\left\|T_{n}\right\|_{\mathcal{P}}$.

Proof of Theorem 2.7. Define $P_{n}: Y \rightarrow Y$ by $P_{n}=\sum_{k=1}^{m_{n}} y_{k, n}^{*} \otimes y_{k, n}$. Then $P_{n}$ is a projection with $\operatorname{ran} P_{n}=F_{n}$ and $\left\|P_{n}\right\|=1$. In fact,

$$
\left\|P_{n} y\right\|=\max _{1 \leq k \leq m_{n}}\left|y_{k, n}^{*}(y)\right| \leq\|y\| \text {. }
$$


Since we also have $Y=\overline{\cup_{n=0}^{\infty} \operatorname{ran} P_{n}}$ and $\operatorname{ran} P_{m} \subset \operatorname{ran} P_{n}$ for $m \leq n$, the following conditions hold:

$$
P_{n} P_{m}=P_{m} \text { for } m \leq n \quad \text { and } \quad P_{n} y \rightarrow y \text { for } y \in Y .
$$

Since

$$
\left\|T P_{n}\right\|_{\mathcal{P}} \leq\|T\|_{\mathcal{P}}\left\|P_{n}\right\|=\|T\|_{\mathcal{P}}
$$

for all $n$ and $T P_{n} y \rightarrow T y$ for all $y \in Y$, it follows from Lemma 2.8 that

$$
\|T\|_{\mathcal{P}} \leq \sup _{n}\left\|T P_{n}\right\|_{\mathcal{P}}
$$

But from $P_{n} P_{m}=P_{m}$ when $m \leq n$, we get

$$
\|T\|_{\mathcal{P}}=\sup _{n}\left\|T P_{n}\right\|_{\mathcal{P}}=\lim _{n}\left\|T P_{n}\right\|_{\mathcal{P}}
$$

We have $T P_{n}=\sum_{k=1}^{m_{n}} y_{k, n}^{*} \otimes T y_{k, n}$. Hence,

$$
T y=\lim _{n} T P_{n} y=\lim _{n} \sum_{k=1}^{m_{n}} y_{k, n}^{*}(y) T y_{k, n}
$$

for all $y \in Y$. We also get

$$
\left\|T P_{n}\right\|_{\mathcal{P}} \leq\left\|\sum_{k=1}^{m_{n}} y_{k, n}^{*} \otimes T y_{k, n}\right\|_{\mathcal{N}} \leq \sum_{k=1}^{m_{n}}\left\|T y_{k, n}\right\| .
$$

On the other hand,

$$
\sum_{k=1}^{m_{n}}\left\|T y_{k, n}\right\| \leq\|T\|_{\mathcal{P}} \sup _{y^{*} \in B_{Y^{*}}} \sum_{k=1}^{m_{n}}\left|y^{*}\left(y_{k, n}\right)\right|=\|T\|_{\mathcal{P}} \sup _{y^{*} \in B_{F_{n}^{*}}} \sum_{k=1}^{m_{n}}\left|y^{*}\left(y_{k, n}\right)\right| .
$$

Since $\left(y_{k, n}\right)_{k=1}^{m_{n}}$ is an admissible basis in $F_{n}$, we get for any $y^{*} \in B_{F_{n}^{*}}$ that $\sum_{k=1}^{m_{n}}\left|y^{*}\left(y_{k, n}\right)\right| \leq 1$. Hence,

$$
\left\|T P_{n}\right\|_{\mathcal{P}} \leq \sum_{k=1}^{m_{n}}\left\|T y_{k, n}\right\| \leq\|T\|_{\mathcal{P}}
$$

and therefore

$$
\|T\|_{\mathcal{P}}=\lim _{n} \sum_{k=1}^{m_{n}}\left\|T y_{k, n}\right\|
$$

Definition 2.9. Let $Y=\overline{\cup_{n=0}^{\infty} F_{n}}$ be a separable Lindenstrauss space with a structure as in Theorem 2.1 (c) and let $\left(y_{k, n}\right)_{k=1}^{m_{n}}$ be an admissible basis in $F_{n}$, $n=0,1, \ldots$. Let $M_{n}, n=0,1, \ldots$, denote the matrix whose $k$-th row is formed by the coefficients of $y_{k, n}$ in $\left(y_{j, n+1}\right)_{j=1}^{m_{n+1}}$. The matrix $M_{n}$ is of order $m_{n} \times m_{n+1}$. Let $X$ be a Banach space. A system $\left(\left(x_{k, n}\right)_{k=1}^{m_{n}}\right)_{n=0}^{\infty}$ of elements in $X$ is called a tree related to $\left(\left(y_{k, n}\right)_{k=1}^{m_{n}}\right)_{n=0}^{\infty}$ if for all $n=0,1, \ldots$

$$
\left(x_{k, n}\right)_{k=1}^{m_{n}}=M_{n} \cdot\left(x_{j, n+1}\right)_{j=1}^{m_{n+1}}
$$

The corresponding $\ell_{1}$-tree space on $X$ is defined as

$$
\ell_{1}^{\text {tree }}(X)=\left\{\left(z_{n}\right)_{n=0}^{\infty} \in \ell_{\infty}\left(\ell_{1}^{m_{n}}(X)\right): z_{n}=M_{n} \cdot z_{n+1}\right\}
$$


with the norm from $\ell_{\infty}\left(\ell_{1}^{m_{n}}(X)\right)$.

By Definition 2.9, $\left(\left(y_{k, n}\right)_{k=1}^{m_{n}}\right)_{n=0}^{\infty}$ is a tree related to itself, and $\ell_{1}^{\text {tree }}(X)$ is a linear subspace of $\ell_{\infty}\left(\ell_{1}^{m_{n}}(X)\right)$ consisting of all trees in $X$ related to $\left(\left(y_{k, n}\right)_{k=1}^{m_{n}}\right)_{n=0}^{\infty}$. Next, we prove that $\ell_{1}^{\text {tree }}(X)$ is isometrically isomorphic to $\mathcal{P}(Y, X)$, hence $\ell_{1}^{\text {tree }}(X)$ is a closed subspace of $\ell_{\infty}\left(\ell_{1}^{m_{n}}(X)\right)$.

Remark 2.10. A two-trunk tree (introduced and studied in [13]) is precisely a tree related to the system of linear B-splines $\left(\left(y_{k, n}\right)_{k=1}^{2^{n}+1}\right)_{n=0}^{\infty} \subset C[0,1]$ from Example 2.4. And the space $\ell_{1}^{\text {tree }}(X)$ of two-trunk trees from [13] is the corresponding $\ell_{1}^{\text {tree }}(X)$ from Definition 2.9.

Theorem 2.11. Let $X$ be a Banach space. Let $Y=\overline{\cup_{n=0}^{\infty} F_{n}}$ be a separable Lindenstrauss space with a structure as in Theorem 2.1 (c) and let $\left(y_{k, n}\right)_{k=1}^{m_{n}}$ be an admissible basis in $F_{n}$ for $n=0,1, \ldots$ Then $\mathcal{P}(Y, X)$ is isometrically isomorphic to the $\ell_{1}$-tree space $\ell_{1}^{\text {tree }}(X)$ related to $\left(\left(y_{k, n}\right)_{k=1}^{m_{n}}\right)_{n=0}^{\infty}$ by the mapping

$$
T \mapsto\left(\left(T y_{k, n}\right)_{k=1}^{m_{n}}\right)_{n=0}^{\infty}, T \in \mathcal{P}(Y, X) .
$$

The inverse mapping

$$
\left(\left(x_{k, n}\right)_{k=1}^{m_{n}}\right)_{n=0}^{\infty} \mapsto T
$$

is given by

$$
T y=\lim _{n} \sum_{k=1}^{m_{n}} y_{k, n}^{*}(y) x_{k, n}, y \in Y,
$$

where $\left(y_{k, n}^{*}\right)_{k=1}^{m_{n}} \subset B_{Y^{*}}$ are functionals forming a biorthogonal system together with $\left(y_{k, n}\right)_{k=1}^{m_{n}} \subset Y$.

Proof. Due to Theorem 2.7, it remains to show the claim about the inverse mapping. So let $z=\left(\left(x_{k, n}\right)_{k=1}^{m_{n}}\right)_{n=0}^{\infty} \in \ell_{1}^{\text {tree }}(X)$. Define $T_{n}=\sum_{k=1}^{m_{n}} y_{k, n}^{*} \otimes x_{k, n}$. Then

$$
\left\|T_{n}\right\|_{\mathcal{P}} \leq\left\|T_{n}\right\|_{\mathcal{N}} \leq \sum_{k=1}^{m_{n}}\left\|x_{k, n}\right\| \leq\|z\|, \quad n=0,1, \ldots
$$

We want to show that the sequence $\left(T_{n}\right)_{n=0}^{\infty}$ converges pointwise in $\mathcal{L}(Y, X)$. Since the sequence $\left(T_{n}\right)_{n=0}^{\infty}$ is bounded and the functions $y_{k, l}, l=0,1, \ldots, k=$ $1, \ldots, m_{l}$, span a dense subspace of $Y$, it suffices to prove that $\lim _{n} T_{n} y_{k, l}$ exists for every $y_{k, l}$. By the definition of $T_{l}$, we have $T_{l} y_{k, l}=x_{k, l}$ for all $l=0,1, \ldots$ and $k=1, \ldots, m_{l}$. Denote the matrix $M_{l}=\left(m_{k, j}^{l}\right)$, so that

$$
y_{k, l}=\sum_{j=1}^{m_{l+1}} m_{k, j}^{l} y_{j, l+1}
$$

and

$$
x_{k, l}=\sum_{j=1}^{m_{l+1}} m_{k, j}^{l} x_{j, l+1}
$$

for all $l=0,1, \ldots$ and $k=1, \ldots, m_{l}$. Since $T_{l+1} y_{j, l+1}=x_{j, l+1}$, we get

$$
T_{l+1} y_{k, l}=T_{l+1}\left(\sum_{j=1}^{m_{l+1}} m_{k, j}^{l} y_{j, l+1}\right)=\sum_{j=1}^{m_{l+1}} m_{k, j}^{l} x_{j, l+1}=x_{k, l} .
$$


Since $T_{l+2} y_{j, l+1}=x_{j, l+1}$, we have

$$
T_{l+2} y_{k, l}=T_{l+2}\left(\sum_{j=1}^{m_{l+1}} m_{k, j}^{l} y_{j, l+1}\right)=x_{k, l} .
$$

Continuing similarly, we get that for each $n \geq l$

$$
T_{n} y_{k, l}=x_{k, l}, k=1, \ldots, m_{l} .
$$

Hence, $\lim _{n} T_{n} y_{k, l}=x_{k, l}$ for all $l=0,1, \ldots$ and $k=1, \ldots, m_{l}$. It follows that $\left(T_{n}\right)_{n=0}^{\infty}$ converges pointwise to an operator $T \in \mathcal{L}(Y, X)$. By Lemma 2.8, $T \in$ $\mathcal{P}(Y, X)$ and $T \mapsto z$ because $T y_{k, l}=x_{k, l}$.

Remark 2.12. Theorems 2.7 and 2.11 can be applied to all Examples above. For instance, one can calculate $\|T\|_{\mathcal{P}}$ for $T \in \mathcal{P}(Y, X)$ using the trees $\left(\left(y_{k, n}\right)_{k=1}^{m_{n}}\right)_{n=0}^{\infty}$ described in the Examples. However, for the representation of $T \in \mathcal{P}(Y, X)$, we need to know about functionals on $Y$ forming biorthogonal systems together with trees. Let us indicate below some appropriate systems of such functionals.

In Example 2.2, we have $Y=M \subset M[0,1]$. We may take $y_{k, n}^{*}=\delta_{(k-1) / 2^{n}}$ (Dirac functionals), $k=1, \ldots, 2^{n}$. Then $\left\|y_{k, n}^{*}\right\|=1$ and

$$
y_{k, n}^{*}\left(y_{j, n}\right)=\delta_{k j} .
$$

If we consider $Y=M \subset L_{\infty}[0,1]$, then we may define the biorthogonal functionals $y_{k, n}^{*} \in B_{M^{*}}$ by

$$
y_{k, n}^{*}(y)=2^{n} \int_{\frac{k-1}{2^{n}}}^{\frac{k}{2^{n}}} y(t) d t, y \in M .
$$

In Example 2.3, we have $Y=C(\Delta)$. We may take $y_{1,0}^{*}=\delta_{0 / 3^{0}}, y_{1,1}^{*}=\delta_{0 / 3^{1}}$, $y_{2,1}^{*}=\delta_{2 / 3^{1}}, y_{1,2}^{*}=\delta_{0 / 3^{2}}, y_{2,2}^{*}=\delta_{2 / 3^{2}}, y_{3,2}^{*}=\delta_{6 / 3^{2}}, y_{4,2}^{*}=\delta_{8 / 3^{2}}$, and so on. Then $\left\|y_{k, n}^{*}\right\|=1$ and

$$
y_{k, n}^{*}\left(y_{j, n}\right)=\delta_{k j}, \quad k, j=1, \ldots, 2^{n} .
$$

In Example 2.4, we have $Y=C[0,1]$. In this case we may take $y_{k, n}^{*}=\delta_{(k-1) / 2^{n}}$, $k=1, \ldots, 2^{n}+1$. Then $\left\|y_{k, n}^{*}\right\|=1$ and

$$
y_{k, n}^{*}\left(y_{j, n}\right)=g_{j-1,2^{n}}\left(\frac{k-1}{2^{n}}\right)=\delta_{j k} .
$$

In this special case Theorem 2.11 reduces to [13, Theorem 3.2].

In Example 2.5, $Y$ is any separable Lindenstrauss space. In [32], Zippin explicitly defined a sequence of functionals $\left(\phi_{k}\right)_{k=1}^{\infty} \subset \operatorname{ext} B_{Y^{*}}$. It follows from Zippin's results that $\left(\left(y_{k, n}\right)_{k=1}^{n+1},\left(\phi_{k}\right)_{k=1}^{n+1}\right)$ is a biorthogonal system.

\section{The $\lambda$-BAP in terms of Lindenstrauss spaces And of $L_{1}[0,1]$}

In [13, Theorems 1.3 and 1.4], we characterized the $\lambda$-BAP and the weak $\lambda$ BAP in terms of $C[0,1]$. In this section (see Theorems 3.3 and 3.4 below), we shall show that $C[0,1]$ can be replaced by many other spaces and we still obtain characterizations of the $\lambda$-BAP and the weak $\lambda$-BAP. An important feature of these spaces is the failure of the Radon-Nikodým property. 
By a well-known theorem of Stegall [31] (see, e.g., [6, p. 198]), $X^{*}$ has the Radon-Nikodým property if and only if every separable subspace $Y$ of $X$ has a separable dual $Y^{*}$. We shall need a reformulation of this result in terms of ideals. Recall that a closed subspace $Y$ of $X$ is an ideal in $X$ if $Y$ admits a norm-preserving extension operator $\varphi \in \mathcal{L}\left(Y^{*}, X^{*}\right)$ (i.e., $\left(\varphi y^{*}\right)(y)=y^{*}(y)$ and $\left\|\varphi y^{*}\right\|=\left\|y^{*}\right\|$ for all $y^{*} \in Y^{*}$ and $\left.y \in Y\right)$. This is equivalent to the annihilator $Y^{\perp}$ of $Y$ being the kernel of a norm one projection on $X^{*}$.

Proposition 3.1. Let $X$ be a Banach space. Then $X^{*}$ has the Radon-Nikodym property if and only if every separable ideal $Y$ in $X$ has a separable dual $Y^{*}$.

Proof. Due to Stegall's theorem, we only need to prove the "if" part. Let $W$ be a separable subspace in $X$. By a result of Heinrich and Mankiewicz [9] or Sims and Yost [29] (see, e.g., [8, p. 138]), we can find a separable ideal $Y$ in $X$ such that $W \subset Y$. Now, $Y^{*}$ is separable and $W^{*}$ is a quotient space of $Y^{*}$, so $W^{*}$ is separable. Hence, $X^{*}$ has the Radon-Nikodým property.

Proposition 3.2. Let $Z$ be a Lindenstrauss space such that $Z^{*}$ fails the RadonNikodym property. Then $Z$ is isometrically universal for all separable Banach spaces.

Proof. By Proposition 3.1, there exists a separable ideal $Y$ in $Z$ such that $Y^{*}$ is not separable. Since $Y$ is an ideal in a Lindenstrauss space, it is also a Lindenstrauss space (see [7, Proposition 3.4]). Now since $Y^{*}$ is non-separable, by a result of Lazar and Lindenstrauss (see [12, Theorem 2.3] or [19, Proposition II.4.18]), $C(\Delta)$ embeds isometrically in $Y$. Since $C(\Delta)$ is isometrically universal for all separable spaces the result follows.

Theorem 3.3. Let $X$ be a Banach space and let $\lambda \in[1, \infty)$. Let $Z$ be a Lindenstrauss space whose dual space $Z^{*}$ fails the Radon-Nikodym property. Then the following statements are equivalent.

(a) $X$ has the $\lambda$-BAP.

(b) For every $T \in \mathcal{I}\left(X, Z^{*}\right)$ there exists a net $\left(S_{\alpha}\right) \subset \mathcal{F}(X, X)$ such that $S_{\alpha} \rightarrow I_{X}$ pointwise and

$$
\limsup _{\alpha}\left\|T S_{\alpha}\right\|_{\mathcal{I}} \leq \lambda\|T\|_{\mathcal{I}}
$$

(c) For every $T \in \mathcal{I}\left(X, L_{1}[0,1]\right)$ there exists a net $\left(S_{\alpha}\right) \subset \mathcal{F}(X, X)$ such that $S_{\alpha} \rightarrow I_{X}$ pointwise and

$$
\limsup \left\|T S_{\alpha}\right\|_{\mathcal{I}} \leq \lambda\|T\|_{\mathcal{I}}
$$

Theorem 3.4. Let $X$ be a Banach space and let $\lambda \in[1, \infty)$. Let $Z$ be a Lindenstrauss space whose dual space $Z^{*}$ fails the Radon-Nikodym property. Then the following statements are equivalent.

(a) $X$ has the weak $\lambda-B A P$.

(b) For every $T \in \mathcal{N}\left(X, Z^{*}\right)$ there exists a net $\left(S_{\alpha}\right) \subset \mathcal{F}(X, X)$ such that $S_{\alpha} \rightarrow I_{X}$ pointwise and

$$
\lim \sup \left\|T S_{\alpha}\right\|_{\mathcal{N}} \leq \lambda\|T\|_{\mathcal{N}}
$$


(c) For every $T \in \mathcal{N}\left(X, L_{1}[0,1]\right)$ there exists a net $\left(S_{\alpha}\right) \subset \mathcal{F}(X, X)$ such that $S_{\alpha} \rightarrow I_{X}$ pointwise and

$$
\limsup _{\alpha}\left\|T S_{\alpha}\right\|_{\mathcal{N}} \leq \lambda\|T\|_{\mathcal{N}}
$$

Remark 3.5. Theorems 1.3 and 1.4 in [15] assert that the equivalences (a) $\Leftrightarrow(b)$ of Theorems 3.3 and 3.4 hold in the particular case when $Z=C[0,1]$. In the above characterizations of the $\lambda$-BAP and the weak $\lambda$-BAP, one may, e.g., take $Z$ to be any separable Lindenstrauss space whose dual space is non-separable, in particular, one may take $Z=M$ or $Z=C(\Delta)$. Comparing characterizations (b) and (c) of the weak BAP and the BAP in Theorems 3.3 and 3.4, it seems to be significant that $L_{1}[0,1]$ is a rather "small" space which is not even a dual space. In (c) of Theorem 3.4, $L_{1}[0,1]$ can be replaced by even a much "smaller" space $\ell_{1}$ (see [14, Proposition 4.1]).

In the proof of Theorem 3.3 we shall use the following lemma.

Lemma 3.6. Let $X$ be a Banach space, let $Y \subset X$ be an ideal, and let $\lambda \in[1, \infty)$. If for every $T \in \mathcal{I}\left(X, L_{1}[0,1]\right)$ there exists a net $\left(S_{\alpha}\right) \subset \mathcal{F}(X, X)$ such that $S_{\alpha} \rightarrow I_{X}$ pointwise on $X$ and

$$
\limsup \left\|T S_{\alpha}\right\|_{\mathcal{I}} \leq \lambda\|T\|_{\mathcal{I}}
$$

then for every $T \in \mathcal{I}\left(Y, L_{1}[0,1]\right)$ there exists a net $\left(S_{\alpha}\right) \subset \mathcal{F}(Y, Y)$ such that $S_{\alpha} \rightarrow I_{Y}$ pointwise on $Y$ and

$$
\limsup _{\alpha}\left\|T S_{\alpha}\right\|_{\mathcal{I}} \leq \lambda\|T\|_{\mathcal{I}}
$$

Proof. Let $T \in \mathcal{I}\left(Y, L_{1}[0,1]\right)$, let $\varphi: Y^{*} \rightarrow X^{*}$ be a norm-preserving extension operator, and let $i_{Y}: Y \rightarrow X$ be the natural embedding. Since integral operators are weakly compact, we have $T^{* *}=j_{L_{1}[0,1]} t$, where $t$ denotes $T^{* *}$ considered with values in $L_{1}[0,1]$. Then (see, e.g., [28, p. 65]) $t \in \mathcal{I}\left(Y^{* *}, L_{1}[0,1]\right)$ and $\|t\|_{\mathcal{I}}=\left\|j_{L_{1}[0,1]} t\right\|_{\mathcal{I}}=\left\|T^{* *}\right\|_{\mathcal{I}}=\|T\|_{\mathcal{I}}$.

Let $F \subset Y$ be a finite set and let $\varepsilon>0$. We have $\left.t \varphi^{*}\right|_{X} \in \mathcal{I}\left(X, L_{1}[0,1]\right)$, so there exists $S \in \mathcal{F}(X, X)$ with $\|S y-y\|<\varepsilon$ for all $y \in F$ and

$$
\left\|t \varphi^{*} S\right\|_{\mathcal{I}} \leq(\lambda+\varepsilon)\left\|t \varphi^{*} j_{X}\right\|_{\mathcal{I}} \leq(\lambda+\varepsilon)\|T\|_{\mathcal{I}} .
$$

Since $\left\|t \varphi^{*} S i_{Y}\right\|_{\mathcal{I}} \leq\left\|t \varphi^{*} S\right\|_{\mathcal{I}}$, we may (simply renaming $S i_{Y}$ to $S$ ) assume that $S \in \mathcal{F}(Y, X)$. All we need to show is that there exists $V \in \mathcal{F}(Y, Y)$ such that $\|V y-y\| \leq \varepsilon$ for all $y \in F$ and

$$
\|T V\|_{\mathcal{I}} \leq(1+\varepsilon)\left\|t \varphi^{*} S\right\|_{\mathcal{I}},
$$

because then also

$$
\|T V\|_{\mathcal{I}} \leq(1+\varepsilon)(\lambda+\varepsilon)\|T\|_{\mathcal{I}}
$$

It is known (see, e.g., [28, p. 176]) that for a finite rank operator, acting to a space with the 1-BAP, its integral norm coincides with its projective tensor norm \|\|$_{\pi}$. Hence, $\|T V\|_{\mathcal{I}}=\|T V\|_{\pi}$ and $\left\|t^{*} \varphi^{*} S\right\|_{\mathcal{I}}=\left\|t \varphi^{*} S\right\|_{\pi}$ in $Y^{*} \hat{\otimes}_{\pi} L_{1}[0,1]$.

Denote

$$
C=\{T V: V \in \mathcal{F}(Y, Y),\|V y-y\| \leq \varepsilon, \forall y \in F\} \subset Y^{*} \otimes L_{1}[0,1]
$$


and

$$
B=(1+\varepsilon)\left\|t \varphi^{*} S\right\|_{\pi} B_{Y^{*} \hat{\otimes}_{\pi} L_{1}[0,1]} .
$$

We need to show that $C \cap B \neq \emptyset$. Observe that $C$ is convex and not empty (take, e.g., any projection $V \in \mathcal{F}(Y, Y)$ onto $\operatorname{span}(F))$.

If $C \cap B=\emptyset$, then there exists $U \in\left(Y^{*} \hat{\otimes}_{\pi} L_{1}[0,1]\right)^{*}=\mathcal{L}\left(Y^{*}, L_{1}[0,1]^{*}\right)$ with $\|U\|=1$ such that

$$
\inf _{T V \in C}\langle U, T V\rangle \geq(1+\varepsilon)\left\|t \varphi^{*} S\right\|_{\pi}
$$

Let $S=\sum_{i=1}^{m} y_{i}^{*} \otimes x_{i}, E=\operatorname{span}\left(F,\left(x_{i}\right)_{i=1}^{m}\right) \subset X$, and $H=\operatorname{span}\left(T^{*} U y_{i}^{*}\right)_{i=1}^{m} \subset$ $Y^{*}$. Choose $\eta>0$ such that $\|S y-y\|<(1+\eta)^{-1} \varepsilon$ for all $y \in F$. Using a local characterization of ideals (see, e.g., [26, Corollary 3.3]), there exists an operator $\psi: E \rightarrow Y$ with $\|\psi\| \leq 1+\eta$ such that $\psi y=y$ for $y \in E \cap Y$ and $y^{*}(\psi x)=\left(\varphi y^{*}\right)(x)$ for all $y^{*} \in H$ and $x \in E$.

Define $V_{\psi}=\sum_{i=1}^{m} y_{i}^{*} \otimes \psi x_{i} \in \mathcal{F}(Y, Y)$. Then $V_{\psi}=\psi S$ and for $y \in F$ we get

$$
\left\|V_{\psi} y-y\right\|=\|\psi S y-\psi y\| \leq(1+\eta)\|S y-y\|<\varepsilon .
$$

Hence, $T V_{\psi} \in C$ and therefore

$$
\begin{aligned}
(1+\varepsilon)\left\|t \varphi^{*} S\right\|_{\pi} & \leq\left\langle U, T V_{\psi}\right\rangle=\sum_{i=1}^{m}\left(U y_{i}^{*}\right)\left(T \psi x_{i}\right)=\sum_{i=1}^{m}\left(\varphi T^{*} U y_{i}^{*}\right)\left(x_{i}\right) \\
& \left.=\sum_{i=1}^{m}\left(U y_{i}^{*}\right)\left(t \varphi^{*} x_{i}\right)\right)=\left\langle U, t \varphi^{*} S\right\rangle \leq\left\|t \varphi^{*} S\right\|_{\pi}
\end{aligned}
$$

which is a contradiction.

Proof of Theorems 3.3 and 3.4. The implications $(\mathrm{a}) \Rightarrow(\mathrm{b})$ and $(\mathrm{a}) \Rightarrow(\mathrm{c})$ hold by [14].

$(b) \Rightarrow(a)$. An examination of the proofs of Theorems 1.3 and $1.4,(b) \Rightarrow(a)$, in $[15]$ reveals that they go through if $C[0,1]$ is replaced by any Banach space $Z$ which is isometrically universal for all separable Banach spaces and such that $Z^{*}$ has the 1-BAP. By Proposition 3.2, $Z$ is isometrically universal for all separable Banach spaces. $Z^{*}$ being an $L_{1}(\mu)$ space, has the 1 -BAP.

(c) $\Rightarrow(\mathrm{a})$. 1. We shall show that if (c) of Theorem 3.3 is satisfied, then for every $T \in \mathcal{I}\left(X, C[0,1]^{*}\right)$ there exists a net $\left(S_{\alpha}\right) \subset \mathcal{F}(X, X)$ such that $S_{\alpha} \rightarrow I_{X}$ pointwise and $\lim \sup _{\alpha}\left\|T S_{\alpha}\right\|_{\mathcal{I}} \leq \lambda\|T\|_{\mathcal{I}}$. Then [15, Theorem 1.3] or Theorem 3.3, (b) $\Rightarrow$ (a), will give the result.

By using Lemma 3.6 and [15, Theorem 3.1] (which is a reformulation of [14, Proposition 4.3 and Theorem 2.2] and asserts that $X$ has the $\lambda$-BAP if and only if every separable ideal of $X$ has the $\lambda$-BAP), we can assume that $X$ is separable.

Let $T \in \mathcal{I}\left(X, C[0,1]^{*}\right)$, let $F$ be a finite subset of $X$, and let $\varepsilon>0$. By definition (see, e.g., [5, pp. 95, 97]), there is a factorization

$$
X \stackrel{b}{\longrightarrow} L_{\infty}(\nu) \stackrel{i_{1}}{\longrightarrow} L_{1}(\nu) \stackrel{a}{\longrightarrow} C[0,1]^{*}
$$

such that $T=a i_{1} b,\|a\|=1$, and $\|b\|<\|T\|_{\mathcal{I}}+\varepsilon$ for some probability measure $\nu$ on $B_{X^{*}}$. Since $X$ is separable, $B_{X^{*}}$ is a separable metric space in the weak* topology. Thus we may assume that $L_{1}(\nu)$ is separable (see, e.g., [1, p. 102]). But then $L_{1}(\nu)$ is linearly isometric to $\ell_{1}(\Gamma) \oplus_{1} L_{1}[0,1]$, where $\Gamma$ is at most countable 
(see [10, p. 128]). Thus there exists an isometry (into) $\psi: L_{1}(\nu) \rightarrow L_{1}[0,1]$. The image $\psi\left(L_{1}(\nu)\right)$ is an $L_{1}$-space, hence it is complemented by a norm one projection $R$ (see [10, p. 162]).

We have $\psi i_{1} b: \mathcal{I}\left(X, L_{1}[0,1]\right)$. Suppose $S \in \mathcal{F}(X, X)$ with $\|S x-x\| \leq \varepsilon$ for all $x \in F$ and $\left\|\psi i_{1} b S\right\|_{\mathcal{I}} \leq(\lambda+\varepsilon)\left\|\psi i_{1} b\right\|_{\mathcal{I}}$. Since $\psi^{-1} R \psi$ is the identity, we get

$$
\begin{aligned}
\|T S\|_{\mathcal{I}} & \leq\left\|i_{1} b S\right\|_{\mathcal{I}}=\left\|\psi^{-1} R \psi i_{1} b S\right\|_{\mathcal{I}} \leq\left\|\psi^{-1} R\right\|\left\|\psi i_{1} b S\right\|_{\mathcal{I}} \\
& \leq(\lambda+\varepsilon)\left\|\psi i_{1} b\right\|_{\mathcal{I}} \leq(\lambda+\varepsilon)\|b\| \leq(\lambda+\varepsilon)\left(\|T\|_{\mathcal{I}}+\varepsilon\right)
\end{aligned}
$$

which is all we need.

2. For the proof of $(\mathrm{c}) \Rightarrow$ (a) in Theorem 3.4, we shall show that if (c) is satisfied, then for every $T \in \mathcal{N}\left(X, \ell_{1}\right)$ there exists a net $\left(S_{\alpha}\right) \subset \mathcal{F}(X, X)$ such that $S_{\alpha} \rightarrow I_{X}$ pointwise and $\lim \sup _{\alpha}\left\|T S_{\alpha}\right\|_{\mathcal{N}} \leq \lambda\|T\|_{\mathcal{N}}$. Then [14, Proposition 4.1] will give (a).

Let $T \in \mathcal{N}\left(X, \ell_{1}\right)$. It is well known that $L_{1}[0,1]$ contains a one-complemented copy of $\ell_{1}$. Let $\psi: \ell_{1} \rightarrow L_{1}[0,1]$ be an isometry (into) and let $R$ be a norm one projection onto $\psi\left(\ell_{1}\right)$. We have $\psi T \in \mathcal{N}\left(X, L_{1}[0,1]\right)$. Let $\left(S_{\alpha}\right) \subset \mathcal{F}(X, X)$ be a net for $\psi T$ as in (c). Since $T=\psi^{-1} R \psi T$,

$$
\limsup _{\alpha}\left\|T S_{\alpha}\right\|_{\mathcal{N}} \leq \limsup _{\alpha}\left\|\psi T S_{\alpha}\right\|_{\mathcal{N}} \leq \lambda\|\psi T\|_{\mathcal{N}} \leq \lambda\|T\|_{\mathcal{N}}
$$

as needed.

3. Let us remark that the proof of $(\mathrm{c}) \Rightarrow(\mathrm{a})$ in Theorem 3.4 can also be done similarly to the proof of $(\mathrm{c}) \Rightarrow(\mathrm{a})$ in Theorem 3.3 by factoring $T \in \mathcal{N}\left(X, C[0,1]^{*}\right)$ through $\ell_{\infty}$ and $\ell_{1}$, and then using that $\ell_{1}$ is isometric to a subspace of $L_{1}[0,1]$.

Indeed, let $T \in \mathcal{N}\left(X, C[0,1]^{*}\right)$ and $\varepsilon>0$. It is well known (see, e.g., [5, p. 111]) that there is a factorization

$$
X \stackrel{b}{\longrightarrow} \ell_{\infty} \stackrel{M_{\lambda}}{\longrightarrow} \ell_{1} \stackrel{a}{\longrightarrow} C[0,1]^{*}
$$

such that $T=a M_{\lambda} b,\|a\|=1,\left\|M_{\lambda}\right\|_{\mathcal{N}}=1$, and $\|b\|<\|T\|_{\mathcal{N}}+\varepsilon$.

We have $\psi M_{\lambda} b \in \mathcal{N}\left(X, L_{1}[0,1]\right)$, where $\psi: \ell_{1} \rightarrow L_{1}[0,1]$ is an into isometry. An argument similar to the argument we used above in the proof of Theorem 3.3 completes the proof.

Concerning Theorems 3.3 and 3.4 and other characterizations of the $\lambda$-BAP and the weak $\lambda$-BAP (see, e.g., [14], [15], [13], [17], [23]), we should add that by [22] (see [25] for a simple proof), the weak $\lambda$-BAP and the $\lambda$-BAP are equivalent for a Banach space $X$ whenever $X^{*}$ or $X^{* *}$ has the Radon-Nikodým property. It remains open whether the weak $\lambda$-BAP is strictly weaker than the $\lambda$-BAP. If they were equivalent, then, by [16], the answer to the long-standing famous open problem (Problem 3.8 in [2]), whether the AP of a dual Banach space implies the 1-BAP, would be "yes". For a recent survey on bounded approximation properties, see [24].

It is well known that a Banach space $X$ has the Radon-Nikodým property if $\mathcal{I}(C[0,1], X)=\mathcal{N}(C[0,1], X)$ (as sets) (see, e.g., [3, p. 523]). And, $X^{*}$ has the Radon-Nikodým property if $\mathcal{I}\left(X, L_{1}[0,1]\right)=\mathcal{N}\left(X, L_{1}[0,1]\right)$ (as sets) (see, e.g., [3, p. 524]). Our Theorem 3.8 below shows that the Radon-Nikodým property can 
be tested for by other single spaces than $C[0,1]$ (for the Radon-Nikodým property of $X$ ) or $L_{1}[0,1]$ (for the Radon-Nikodým property of $X^{*}$ ).

Lemma 3.7. Let $X$ and $Y$ be Banach spaces. If $\mathcal{I}(X, Y)=\mathcal{N}(X, Y)$ (as sets) and $Z$ is an ideal in $X$, then $\mathcal{I}(Z, Y)=\mathcal{N}(Z, Y)$ (as sets).

Proof. Let $\varphi: Z^{*} \rightarrow X^{*}$ be a norm-preserving extension operator and let $T \in$ $\mathcal{I}(Z, Y)$. Since integral operators are weakly compact, we have (using properties of integral operators as in the proof of Lemma 3.6) $\left.T^{* *} \varphi^{*}\right|_{X} \in \mathcal{I}(X, Y)=$ $\mathcal{N}(X, Y)$. Write $T^{* *} \varphi^{*} x=\sum_{n} x_{n}^{*}(x) y_{n}, x \in X$, where $\sum_{n}\left\|x_{n}^{*}\right\|\left\|y_{n}\right\|<\infty$. Then for all $z \in Z$ we get

$$
T z=T^{* *} \varphi^{*} z=\sum_{n} x_{n}^{*}(z) y_{n}=\left.\sum_{n} x_{n}^{*}\right|_{Z}(z) y_{n}
$$

Thus $T=\left.\sum_{n} x_{n}^{*}\right|_{Z} \otimes y_{n} \in \mathcal{N}(Z, Y)$.

Theorem 3.8. Let $X$ be a Banach space and let $Z$ be a Lindenstrauss space whose dual $Z^{*}$ fails the Radon-Nikodým property.

(a) If $\mathcal{I}(Z, X)=\mathcal{N}(Z, X)$ (as sets), then $X$ has the Radon-Nikodym property.

(b) If $\mathcal{I}\left(X, Z^{*}\right)=\mathcal{N}\left(X, Z^{*}\right)$ (as sets), then $X^{*}$ has the Radon-Nikodym property.

Proof. (a) By Proposition 3.2, $C[0,1] \subset Z$. But any Lindenstrauss space is an ideal in every "superspace" (see [7, Proposition 3.4]), in particular, $C[0,1]$ is an ideal in $Z$. Since $\mathcal{I}(Z, X)=\mathcal{N}(Z, X)$, by Lemma 3.7, also $\mathcal{I}(C[0,1], X)=$ $\mathcal{N}(C[0,1], X)$. Hence, $X$ has the Radon-Nikodým property.

(b) This follows when we apply (a) to $X^{*}$. Indeed, let $T \in \mathcal{I}\left(Z, X^{*}\right)$. Then $T^{*} \in$ $\mathcal{I}\left(X^{* *}, Z^{*}\right)$ and $T^{*} j_{X} \in \mathcal{I}\left(X, Z^{*}\right)=\mathcal{N}\left(X, Z^{*}\right)$. Hence, $\left(j_{X}\right)^{*} T^{* *} \in \mathcal{N}\left(Z^{* *}, X^{*}\right)$ and $\left(j_{X}\right)^{*} T^{* *} j_{Z} \in \mathcal{N}\left(Z, X^{*}\right)$. But $\left(j_{X}\right)^{*} T^{* *} j_{Z}=\left(j_{X}\right)^{*} j_{X^{*}} T=T$.

\section{The Spaces $\mathcal{I}\left(X, Z^{*}\right)$, With a Lindenstrauss spaCe $Z$, And $\mathcal{I}\left(X, L_{1}[0,1]\right)$}

Let $X$ be a Banach space and let $Z$ be a Lindenstrauss space. In Theorems 3.3 and 3.4, we characterized the $\lambda$-BAP and the weak $\lambda$-BAP of $X$ in terms of $\mathcal{I}\left(X, Z^{*}\right)$ and $\mathcal{I}\left(X, L_{1}[0,1]\right)$, and of $\mathcal{N}\left(X, Z^{*}\right)$ and $\mathcal{N}\left(X, L_{1}[0,1]\right)$, respectively. In particular, the corresponding norms of operators were used. It is rather well known how to calculate nuclear norms in the latter spaces, since $\mathcal{N}\left(X, Z^{*}\right)=$ $\mathcal{N}\left(X, L_{1}(\mu)\right)=X^{*} \hat{\otimes}_{\pi} L_{1}(\mu)=L_{1}\left(\mu, X^{*}\right)$, an $X^{*}$-valued Lebesgue-Bochner space for some measure $\mu$, and similarly, $\mathcal{N}\left(X, L_{1}[0,1]\right)=L_{1}\left([0,1], X^{*}\right)$ (see e.g., [28, pp. 76, 29]). This seems not to be the case for the former spaces. In this section, applying results and ideas from Sections 2 and 3, we shall look at the structure of the spaces $\mathcal{I}\left(X, Z^{*}\right)$ and $\mathcal{I}\left(X, L_{1}[0,1]\right)$. In particular, we shall indicate formulas for computing respective integral norms.

4.1. Computing norm in $\mathcal{I}\left(X, Z^{*}\right)$. Let $X$ and $Z$ be Banach spaces. Using basic properties of integral operators (see, e.g., [28, p. 65]), it is straightforward 
to verify that $\mathcal{I}\left(X, Z^{*}\right)$ is isometrically isomorphic to $\mathcal{I}\left(Z, X^{*}\right)$ by the mapping $T \mapsto T^{*} j_{Z}$. Indeed,

$$
\left\|T^{*} j_{Z}\right\|_{\mathcal{I}} \leq\left\|T^{*}\right\|_{\mathcal{I}}=\|T\|_{\mathcal{I}}=\left\|j_{Z}^{*} T^{* *} j_{X}\right\|_{\mathcal{I}} \leq\left\|j_{Z}^{*} T^{* *}\right\|_{\mathcal{I}}=\left\|T^{*} j_{Z}\right\|_{\mathcal{I}}
$$

meaning that $\left\|T^{*} j_{Z}\right\|_{\mathcal{I}}=\|T\|_{\mathcal{I}}$ for all $T \in \mathcal{I}\left(X, Z^{*}\right)$. On the other hand, if $S \in \mathcal{I}\left(Z, X^{*}\right)$, then

$$
S=j_{X}^{*} S^{* *} j_{Z}=\left(S^{*} j_{X}\right)^{*} j_{Z}
$$

In the case when $Z$ is a Lindenstrauss space, by a result of Stegall [30], one has $\mathcal{I}(Z, X)=\mathcal{P}(Z, X)$ as Banach spaces. Hence, the following is immediate from Theorems 2.7 and 2.11 .

Theorem 4.1. Let $X$ be a Banach space. Let $Z=\overline{\cup_{n=0}^{\infty} F_{n}}$ be a separable Lindenstrauss space with a structure as in Theorem 2.1 (c) and let $\left(y_{k, n}\right)_{k=1}^{m_{n}}$ be an admissible basis in $F_{n}$ for $n=0,1, \ldots$ Then $\mathcal{I}\left(X, Z^{*}\right)$ is isometrically isomorphic to the $\ell_{1}$-tree space $\ell_{1}^{\text {tree }}\left(X^{*}\right)$ related $\left(\left(y_{k, n}\right)_{k=1}^{m_{n}}\right)_{n=0}^{\infty}$ by the mapping

$$
T \mapsto\left(\left(T^{*} y_{k, n}\right)_{k=1}^{m_{n}}\right)_{n=0}^{\infty}, T \in \mathcal{I}\left(X, Z^{*}\right)
$$

and

$$
\|T\|_{\mathcal{I}}=\sup _{n} \sum_{k=1}^{m_{n}}\left\|T^{*} y_{k, n}\right\|=\lim _{n} \sum_{k=1}^{m_{n}}\left\|T^{*} y_{k, n}\right\| .
$$

In Theorem 4.7 below, we shall deduce a similar formula for $\|T\|_{\mathcal{I}}$ when $T \in$ $\mathcal{I}\left(X, L_{1}[0,1]\right)$.

4.2. $\mathcal{I}\left(X, L_{1}[0,1]\right)$ as an $L$-summand. The first result concerning the structure of $\mathcal{I}\left(X, L_{1}[0,1]\right)$, Theorem 4.5 , is that it is an $L$-summand in $\mathcal{I}\left(X, C[0,1]^{*}\right)$. The proof relies on a corresponding structure result on $\mathcal{I}(Z, X)=\mathcal{P}(Z, X)$, where $Z$ is a Lindenstrauss space (see Theorem 4.3).

From the definition of the absolute summing norm the following result follows.

Lemma 4.2. Let $X$ and $Z$ be Banach spaces and let $S \in \mathcal{P}(Z, X)$. Then there exists a separable subspace $Y \subset Z$ such that $\|S\|_{\mathcal{P}}=\left\|\left.S\right|_{Y}\right\|_{\mathcal{P}}$.

Theorem 4.3. Let $Z$ be a Lindenstrauss space and let $X$ be a Banach space. If $T \in \mathcal{P}(Z, X)$ and $P$ is an L-projection on $X$, then

$$
\|T\|_{\mathcal{P}}=\|P T\|_{\mathcal{P}}+\|(I-P) T\|_{\mathcal{P}}
$$

Proof. The inequality $\|T\|_{\mathcal{P}} \leq\|P T\|_{\mathcal{P}}+\|(I-P) T\|_{\mathcal{P}}$ is trivial. In order to prove the converse, by the lemma above, there exists a separable subspace $Y \subset Z$ such that $\|P T\|_{\mathcal{P}}=\left\|\left.P T\right|_{Y}\right\|_{\mathcal{P}}$ and $\|(I-P) T\|_{\mathcal{P}}=\left\|\left.(I-P) T\right|_{Y}\right\|_{\mathcal{P}}$. As in the proof of Proposition 3.1, we may assume that $Y$ is an ideal in $Z$. But then $Y$ is a separable Lindenstrauss space.

As in Theorem 2.7, we may choose a sequence of admissible bases $\left(\left(y_{k, n}\right)_{k=1}^{m_{n}}\right)_{n=0}^{\infty}$ for $Y=\overline{\cup_{n=0}^{\infty} F_{n}}$. Then, by Theorem 2.7, we get

$$
\begin{aligned}
\|T\|_{\mathcal{P}} \geq\left\|\left.T\right|_{Y}\right\|_{\mathcal{P}} & =\lim _{n} \sum_{k=1}^{m_{n}}\left\|T y_{k, n}\right\|=\lim _{n}\left(\sum_{k=1}^{m_{n}}\left\|P T y_{k, n}\right\|+\sum_{k=1}^{m_{n}}\left\|(I-P) T y_{k, n}\right\|\right) \\
& =\left\|\left.P T\right|_{Y}\right\|_{\mathcal{P}}+\left\|\left.(I-P) T\right|_{Y}\right\|_{\mathcal{P}}=\|P T\|_{\mathcal{P}}+\|(I-P) T\|_{\mathcal{P}} .
\end{aligned}
$$


Proposition 4.4. Let $X$ and $Y$ be Banach spaces and assume that $Y$ is onecomplemented in its bidual. Let $P$ be an L-projection on $Y$. Then

$$
\|T\|_{\mathcal{I}}=\|P T\|_{\mathcal{I}}+\|(I-P) T\|_{\mathcal{I}}
$$

for every $T \in \mathcal{I}(X, Y)$.

Proof. Let $T \in \mathcal{I}(X, Y)$ and let $\varepsilon>0$. Since $Y$ is one-complemented in its bidual, by [6, p. 235], $T$ is Pietsch integral. By [6, p. 168], $T$ admits a factorization through a $C(K)$ space, where $K$ is compact Hausdorff. That is, for $\varepsilon>0$ there exist a norm one operator $R: X \rightarrow C(K)$ and an absolutely summing operator $S: C(K) \rightarrow Y$ such that $T=S R$ and $\|T\|_{\mathcal{I}} \leq\|S\|_{\mathcal{P}} \leq\|T\|_{\mathcal{I}}+\varepsilon$. Since $\mathcal{P}(C(K), Y)=\mathcal{I}(C(K), Y)$ with equal norms (see [6, pp. 169, 235]), from Theorem 4.3 we get

$$
\|S\|_{\mathcal{P}}=\|P S\|_{\mathcal{P}}+\|(I-P) S\|_{\mathcal{P}}=\|P S\|_{\mathcal{I}}+\|(I-P) S\|_{\mathcal{I}} .
$$

Hence,

$$
\begin{aligned}
\|T\|_{\mathcal{I}}+\varepsilon & \geq\|S\|_{\mathcal{P}}=\|P S\|_{\mathcal{I}}+\|(I-P) S\|_{\mathcal{I}} \\
& \geq\|P S R\|_{\mathcal{I}}+\|(I-P) S R\|_{\mathcal{I}}=\|P T\|_{\mathcal{I}}+\|(I-P) T\|_{\mathcal{I}}
\end{aligned}
$$

so that $\|T\|_{\mathcal{I}}=\|P T\|_{\mathcal{I}}+\|(I-P) T\|_{\mathcal{I}}$.

The dual space $C[0,1]^{*}$ can be identified with the space of regular Borel measures on $[0,1]$. It is well known that $L_{1}[0,1]$ is an $L$-summand in $C[0,1]^{*}$. This comes from the fact that if $\mu \in C[0,1]^{*}$, then its Lebesgue decomposition $\mu=\mu_{\mathrm{ac}}+\mu_{\text {sing }}$ satisfies $\|\mu\|=\left\|\mu_{\mathrm{ac}}\right\|+\left\|\mu_{\text {sing }}\right\|$. By the Radon-Nikodým theorem, we can write $d \mu_{\mathrm{ac}}=f d t$, where $d t$ is the Lebesgue measure on $[0,1]$ and $f \in L_{1}[0,1]$. And, $\left\|\mu_{\text {ac }}\right\|=\|f\|$. The $L$-projection $P$ onto $L_{1}[0,1]$ is given by $P \mu=f$. By Proposition 4.4, we now can state the following theorem.

Theorem 4.5. Let $X$ be a Banach space. Then $\mathcal{I}\left(X, L_{1}[0,1]\right)$ is an L-summand in $\mathcal{I}\left(X, C[0,1]^{*}\right)$.

4.3. The space $\mathcal{I}\left(X, L_{1}[0,1]\right)$. As in Example 2.2, see Section 2, let

$$
y_{k, n}=\chi_{\left[\frac{k-1}{2^{n}}, \frac{k}{2^{n}}\right)}
$$

for $n=0,1, \ldots$ and $k=1, \ldots, 2^{n}$. While convenient, we consider $y_{k, n}$ as elements of $L_{1}[0,1]$ or of $L_{\infty}[0,1]=L_{1}[0,1]^{*}$.

The main result of this subsection is Theorem 4.7. It gives a reasonable formula for computing $\|T\|_{\mathcal{I}}$ of $T \in \mathcal{I}\left(X, L_{1}[0,1]\right)$ in terms of $y_{k, n} \in L_{\infty}[0,1]$. As a byproduct, we shall also calculate the norm in $\mathcal{L}\left(L_{1}[0,1], X\right)$ (see Theorem 4.6).

Below, we shall use the following notation.

Let $\left(h_{j}\right)_{j=1}^{\infty}$ be the Haar basis in $L_{1}[0,1]$. With the definition as in [4], we have $h_{1}=1$ and, for $n=0,1,2, \ldots$ and $i=1, \ldots, 2^{n}$,

$$
h_{2^{n}+i}=\chi_{\left[\frac{2 i-2}{2^{n+1}}, \frac{2 i-1}{2^{n+1}}\right)}-\chi_{\left[\frac{2 i-1}{2^{n+1}}, \frac{2 i}{2^{n+1}}\right)}=y_{2 i-1, n+1}-y_{2 i, n+1} .
$$

Let $\left(h_{j}^{*}\right)_{j=1}^{\infty}$ denote the coordinate functionals of the Haar basis $\left(h_{j}\right)_{j=1}^{\infty}$.

Denote

$$
W_{n}=\operatorname{span}\left(h_{k}\right)_{k=1}^{2^{n}}=\operatorname{span}\left(y_{k, n}\right)_{k=1}^{2^{n}} \subset L_{1}[0,1]
$$


and let $P_{n}: L_{1}[0,1] \rightarrow W_{n}$ be the natural projection in $L_{1}[0,1]$, associated to the basis $\left(h_{j}\right)_{j=1}^{\infty}$, i.e., $P_{n}=\sum_{j=1}^{2^{n}} h_{j}^{*} \otimes h_{j}$. Since the Haar basis is monotone, we have $\left\|P_{n}\right\|=1$ for $n=0,1,2, \ldots$.

We shall need the following description for the extreme points of $B_{W_{n}}$ :

$$
\operatorname{ext} B_{W_{n}}=\left\{ \pm 2^{n} y_{k, n}: 1 \leq k \leq 2^{n}\right\} .
$$

This comes from the fact that the map $\theta_{n}: W_{n} \rightarrow \ell_{1}^{2^{n}}$ defined by $\theta_{n}\left(2^{n} y_{k, n}\right)=e_{k}$ is a linear isometry and ext $B_{\ell_{1}^{2}}=\left\{ \pm e_{k}: 1 \leq k \leq 2^{n}\right\}$.

Theorem 4.6. Let $V \in \mathcal{L}\left(L_{1}[0,1], X\right)$. Then

$$
\|V\|=\sup _{n \geq 0,1 \leq k \leq 2^{n}}\left\|V\left(2^{n} y_{k, n}\right)\right\|=\lim _{n} \max _{1 \leq k \leq 2^{n}}\left\|V\left(2^{n} y_{k, n}\right)\right\| .
$$

Proof. Let $V \in \mathcal{L}\left(L_{1}[0,1], X\right)$. Then

$$
\left\|V P_{n}\right\| \geq\left\|V P_{n}\left(2^{n} y_{k, n}\right)\right\|=\left\|V\left(2^{n} y_{k, n}\right)\right\| .
$$

On the other hand, using (4.1), we get

$$
\begin{aligned}
\left\|V P_{n}\right\| & =\sup _{\|f\| \leq 1}\left\|V P_{n} f\right\| \leq \sup _{\left\|P_{n} f\right\| \leq 1}\left\|V P_{n} f\right\| \\
& =\left\|\left.V\right|_{W_{n}}\right\|=\sup _{g \in \operatorname{ext} B_{W_{n}}}\|V g\|=\max _{1 \leq k \leq 2^{n}}\left\|V\left(2^{n} y_{k, n}\right)\right\| .
\end{aligned}
$$

Hence,

$$
\left\|V P_{n}\right\|=\max _{1 \leq k \leq 2^{n}}\left\|V\left(2^{n} y_{k, n}\right)\right\| .
$$

For $f \in L_{1}[0,1]$ we have $V P_{n} f \rightarrow V f$. Hence, $\|V\| \leq \sup \left\|V P_{n}\right\|$. Since $\left\|V P_{n}\right\| \leq\left\|V P_{n+1}\right\| \leq\|V\|$, we get

$$
\|V\|=\lim _{n}\left\|V P_{n}\right\|=\sup _{n \geq 0,1 \leq k \leq 2^{n}}\left\|V\left(2^{n} y_{k, n}\right)\right\| .
$$

In the proof of the next theorem we shall use the following simple formula for the projections $\left(P_{n}\right)_{n=0}^{\infty}$ :

$$
P_{n}=\sum_{k=1}^{2^{n}} y_{k, n} \otimes 2^{n} y_{k, n} .
$$

Since $\left(2^{n} y_{k, n}\right)_{k=1}^{2^{n}}$ is a basis of $W_{n}$ and $\left(2^{n} y_{k, n}, y_{k, n}\right)_{k=1}^{2^{n}}\left(\right.$ with $y_{k, n} \in L_{\infty}[0,1]=$ $\left.L_{1}[0,1]^{*}\right)$ is a biorthogonal system,

$$
P_{n} f=\sum_{k=1}^{2^{n}} y_{k, n}\left(P_{n} f\right) 2^{n} y_{k, n}
$$

for all $f \in L_{1}[0,1]$. Hence,

$$
P_{n}=\sum_{k=1}^{2^{n}} P_{n}^{*} y_{k, n} \otimes 2^{n} y_{k, n} .
$$


But $P_{n}^{*} y_{k, n}=y_{k, n}$, because $y_{k, n}\left(h_{j}\right)=0$ whenever $j>2^{n}$, implying that

$$
y_{k, n}(f)=\sum_{j=1}^{2^{n}} h_{j}^{*}(f) y_{k, n}\left(h_{j}\right)=y_{k, n}\left(P_{n} f\right)
$$

for all $f \in L_{1}[0,1]$.

Theorem 4.7. Let $T \in \mathcal{I}\left(X, L_{1}[0,1]\right)$. Then

$$
\|T\|_{\mathcal{I}}=\sup _{n} \sum_{k=1}^{2^{n}}\left\|T^{*} y_{k, n}\right\|=\lim _{n} \sum_{k=1}^{2^{n}}\left\|T^{*} y_{k, n}\right\|
$$

Proof. Let $T \in \mathcal{I}\left(X, L_{1}[0,1]\right) \subset \mathcal{I}\left(X, L_{1}[0,1]^{* *}\right)=\mathcal{F}\left(L_{1}[0,1], X\right)^{*}$. If $V=g^{*} \otimes$ $x \in \mathcal{F}\left(L_{1}[0,1], X\right)$, then $V P_{n}=P_{n}^{*} g^{*} \otimes x$. Since

$$
\langle T, V\rangle=g^{*}(T x)=g^{*}\left(\lim _{n} P_{n} T x\right)=\lim _{n}\left(P_{n}^{*} g^{*}\right)(T x)=\lim _{n}\left\langle T, V P_{n}\right\rangle,
$$

we have

$$
\langle T, V\rangle=\lim _{n}\left\langle T, V P_{n}\right\rangle
$$

for all $V \in \mathcal{F}\left(L_{1}[0,1], X\right)$.

Let $\varepsilon>0$. Choose $V \in \mathcal{F}\left(L_{1}[0,1], X\right)$ with $\|V\| \leq 1$ and choose $n \in \mathbb{N}$ such that

$$
\|T\|_{\mathcal{I}}-\varepsilon<\left\langle T, V P_{n}\right\rangle
$$

By (4.2) we can write

$$
\begin{aligned}
\left\langle T, V P_{n}\right\rangle & =\left\langle T, \sum_{k=1}^{2^{n}} y_{k, n} \otimes V\left(2^{n} y_{k, n}\right)\right\rangle=\sum_{k=1}^{2^{n}}\left(T^{*} y_{k, n}\right)\left(V\left(2^{n} y_{k, n}\right)\right) \\
& \leq\|V\| \sum_{k=1}^{2^{n}}\left\|T^{*} y_{k, n}\right\| \leq \sum_{k=1}^{2^{n}}\left\|T^{*} y_{k, n}\right\| .
\end{aligned}
$$

Thus we get

$$
\|T\|_{\mathcal{I}} \leq \sup _{n} \sum_{k=1}^{2^{n}}\left\|T^{*} y_{k, n}\right\| .
$$

On the other hand, by Theorem 2.7,

$$
\|T\|_{\mathcal{I}}=\left\|T^{*}\right\|_{\mathcal{I}} \geq\left\|T^{*}\right\|_{\mathcal{P}} \geq\left\|\left.T^{*}\right|_{M}\right\|_{\mathcal{P}}=\sup _{n} \sum_{k=1}^{2^{n}}\left\|T^{*} y_{k, n}\right\|=\lim _{n} \sum_{k=1}^{2^{n}}\left\|T^{*} y_{k, n}\right\|,
$$

where $M \subset L_{\infty}[0,1]$ is as in Example 2.3 (see Section 2).

We can write $\left(\left(y_{k, n}\right)_{k=1}^{2^{n}}\right)_{n=0}^{\infty}$ as a sequence $y_{1,0}, y_{1,1}, y_{2,1}, y_{1,2}, \ldots$. Then $y_{k, n}$ is the element of number $2^{n}+k-1$.

Proposition 4.8. For every $T \in \mathcal{I}\left(X, L_{1}[0,1]\right)$,

$$
\lim _{n} \max _{1 \leq k \leq 2^{n}}\left\|T^{*} y_{k, n}\right\|=0 .
$$


Proof. By [5, Theorem 5.19], there exists $g \in L_{1}[0,1]$ such that $T\left(B_{X}\right) \subset[-g, g]$, where $[-g, g]$ is the order interval. We have

$$
\left\|T^{*} y_{k, n}\right\|=\sup _{x \in B_{X}} y_{k, n}(T x) \leq y_{k, n}(g) .
$$

Write $g=\sum_{i=1}^{\infty} a_{i} h_{i}$. Let $g_{m}=\sum_{i=1}^{m} a_{i} h_{i}$. We get $\left|y_{k, n}\left(g-g_{m}\right)\right| \leq\left\|g-g_{m}\right\| \rightarrow_{m}$ 0 . Thus, it suffices to prove that $y_{k, n}\left(g_{m}\right) \rightarrow_{n} 0$ for a fixed $m$.

Fix $m$. If $1 \leq k \leq 2^{n}$, then

$$
\begin{aligned}
\left|y_{k, n}\left(g_{m}\right)\right| & \leq \sum_{i=1}^{m}\left|a_{i}\right| \int_{0}^{1}\left|h_{i}(t)\right|\left|y_{k, n}(t)\right| d t \\
& \leq \sum_{i=1}^{m}\left|a_{i}\right| \int_{0}^{1} y_{k, n}(t) d t=\frac{1}{2^{n}} \sum_{i=1}^{m}\left|a_{i}\right| \rightarrow_{n} 0 .
\end{aligned}
$$

Defining $\hat{h}_{1}=h_{1}$ and $\hat{h}_{n}=2^{m-1} h_{n}$, where $2^{m-1}<n \leq 2^{m}$ and $n \in \mathbb{N}$, one obtains the normalized Haar basis $\left(\hat{h}_{n}\right)_{n=1}^{\infty}$ for $L_{1}[0,1]$. Its coordinate functionals are $\left(h_{n}\right)_{n=1}^{\infty} \subset L_{\infty}[0,1]$.

Lemma 4.9. Let $Y$ be a Banach space with a basis $\left(y_{n}\right)_{n=1}^{\infty}$ and with the coordinate functionals $\left(y_{n}^{*}\right)_{n=1}^{\infty}$. If $T \in \mathcal{I}(X, Y) \subset \mathcal{I}\left(X, Y^{* *}\right)=\mathcal{F}(Y, X)^{*}$ and $V \in \mathcal{F}(Y, X)$, then

$$
\langle T, V\rangle=\sum_{n=1}^{\infty}\left(T^{*} y_{n}^{*}\right)\left(V y_{n}\right)
$$

Proof. It clearly suffices to prove the claim for $V=y^{*} \otimes x \in \mathcal{F}(Y, X)$. Then we get

$$
\begin{aligned}
\langle T, V\rangle & =y^{*}(T x)=y^{*}\left(\sum_{n=1}^{\infty} y_{n}^{*}(T x) y_{n}\right)=\sum_{n=1}^{\infty}\left(T^{*} y_{n}^{*}\right)(x) y^{*}\left(y_{n}\right) \\
& =\sum_{n=1}^{\infty}\left(T^{*} y_{n}^{*}\right)\left(y^{*}\left(y_{n}\right) x\right)=\sum_{n=1}^{\infty}\left(T^{*} y_{n}^{*}\right)\left(V y_{n}\right)
\end{aligned}
$$

Corollary 4.10. Let $T \in \mathcal{I}\left(X, L_{1}[0,1]\right)$ and $V \in \mathcal{F}\left(L_{1}[0,1], X\right)$. Then we have

$$
\langle T, V\rangle=\sum_{n=1}^{\infty}\left(T^{*} h_{n}\right)\left(V \hat{h}_{n}\right)=\sum_{n=1}^{\infty}\left(T^{*} h_{n}^{*}\right)\left(V h_{n}\right) .
$$

Remark 4.11. Note that, in general, $\left\|V \hat{h}_{n}\right\| \nrightarrow 0$ in Corollary 4.10. Indeed, take $V=g^{*} \otimes x$, where $x \in S_{X}$ and $g^{*} \in L_{\infty}[0,1]$ is defined as follows:

$$
g^{*}(t)= \begin{cases}h_{3}(t) & \text { if } t \in[0,1 / 2) \\ h_{7}(t) & \text { if } t \in[1 / 2,3 / 4) \\ h_{15}(t) & \text { if } t \in[3 / 4,7 / 8) \\ \vdots & \text { and so on. }\end{cases}
$$


Then

$$
\left\|V \hat{h}_{2^{m}-1}\right\|=\left|g^{*}\left(\hat{h}_{2^{m}-1}\right)\right|=\int_{0}^{1} g^{*}(t) \hat{h}_{\left(2^{m}-1\right)}(t) d t=\int_{0}^{1} h_{2^{m}-1}(t) \hat{h}_{2^{m}-1}(t) d t=1 .
$$

Hence, $\left\|V \hat{h}_{n}\right\| \nrightarrow 0$ and $\hat{h}_{n} \nrightarrow 0$ weakly.

4.4. The Haar basis and $\mathcal{I}\left(X, L_{1}[0,1]\right)$. Let $n \in \mathbb{N}$. Both $\left(h_{k}\right)_{k=1}^{2^{n}}$ and $\left(y_{k, n}\right)_{k=1}^{2^{n}}$ are bases for $W_{n}$. Thus, there exists a $2^{n} \times 2^{n}$ matrix $C_{n}$ such that

$$
\left(\begin{array}{c}
h_{1} \\
\vdots \\
h_{2^{n}}
\end{array}\right)=C_{n} \cdot\left(\begin{array}{c}
y_{1, n} \\
\vdots \\
y_{2^{n}, n}
\end{array}\right) \text { and }\left(\begin{array}{c}
y_{1, n} \\
\vdots \\
y_{2^{n}, n}
\end{array}\right)=C_{n}^{-1} \cdot\left(\begin{array}{c}
h_{1} \\
\vdots \\
h_{2^{n}}
\end{array}\right) \text {. }
$$

If $i \leq 2^{n}$, then

$$
h_{i}\left(y_{k, n}\right)=\int_{(k-1) / 2^{n}}^{k / 2^{n}} h_{i}(t) d t=\frac{1}{2^{n}} h_{i}\left(\frac{k-1}{2^{n}}\right) .
$$

Thus we get

$$
y_{k, n}=\sum_{i=1}^{2^{n}} \frac{1}{2^{n}} h_{i}\left(\frac{k-1}{2^{n}}\right) \hat{h}_{i}=\sum_{i=1}^{2^{n}} \frac{1}{2^{n}} \hat{h}_{i}\left(\frac{k-1}{2^{n}}\right) h_{i}
$$

It follows that

$$
C_{n}^{-1}=\left(\frac{1}{2^{n}} \hat{h}_{i}\left(\frac{k-1}{2^{n}}\right)\right)
$$

where $k$ is the row number and $i$ is the column number.

In (4.3) we can apply at points $\left(\frac{j-1}{2^{n}}\right)_{j=1}^{2^{n}}$ and we get

$$
\delta_{k j}=y_{k, n}\left(\frac{j-1}{2^{n}}\right)=\sum_{i=1}^{2^{n}} \frac{1}{2^{n}} \hat{h}_{i}\left(\frac{k-1}{2^{n}}\right) h_{i}\left(\frac{j-1}{2^{n}}\right) .
$$

Hence, we get

$$
C_{n}=\left(h_{i}\left(\frac{k-1}{2^{n}}\right)\right),
$$

where $i$ is the row number and $k$ is the column number. Moreover,

$$
h_{i}=\sum_{k=1}^{2^{n}} h_{i}\left(\frac{k-1}{2^{n}}\right) y_{k, n} .
$$

Let us give two examples.

$$
\begin{gathered}
C_{1}=\left(\begin{array}{rr}
1 & 1 \\
1 & -1
\end{array}\right) \quad \text { and } C_{1}^{-1}=\left(\begin{array}{rr}
\frac{1}{2} & \frac{1}{2} \\
\frac{1}{2} & -\frac{1}{2}
\end{array}\right) . \\
C_{2}=\left(\begin{array}{rrrr}
1 & 1 & 1 & 1 \\
1 & 1 & -1 & -1 \\
1 & -1 & 0 & 0 \\
0 & 0 & 1 & -1
\end{array}\right) \quad \text { and } C_{2}^{-1}=\left(\begin{array}{rrrr}
\frac{1}{4} & \frac{1}{4} & \frac{1}{2} & 0 \\
\frac{1}{4} & \frac{1}{4} & -\frac{1}{2} & 0 \\
\frac{1}{4} & -\frac{1}{4} & 0 & \frac{1}{2} \\
\frac{1}{4} & -\frac{1}{4} & 0 & -\frac{1}{2}
\end{array}\right) .
\end{gathered}
$$

From Theorems 4.6 and 4.7 we now get the following formulas which connect the norms of the operators with the entities we used in Corollary 4.10. 
Theorem 4.12. Let $V \in \mathcal{L}\left(L_{1}[0,1], X\right)$ and $T \in \mathcal{I}\left(X, L_{1}[0,1]\right)$. Then

$$
\begin{aligned}
& \|V\|=\lim _{n} \max _{1 \leq k \leq 2^{n}}\left\|\sum_{i=1}^{2^{n}} h_{i}\left(\frac{k-1}{2^{n}}\right) V \hat{h}_{i}\right\|, \text { and } \\
& \|T\|_{\mathcal{I}}=\lim _{n} \frac{1}{2^{n}} \sum_{k=1}^{2^{n}}\left\|\sum_{i=1}^{2^{n}} \hat{h}_{i}\left(\frac{k-1}{2^{n}}\right) T^{*} h_{i}\right\| .
\end{aligned}
$$

Acknowledgement. The research of Eve Oja was partially supported by Estonian Science Foundation Grant 8976 and Estonian Targeted Financing Project SF0180039s08.

\section{REFERENCES}

1. F. Albiac and N. Kalton, Topics in Banach Space Theory, Graduate Texts in Mathematics, 233, Springer-Verlag, 2006.

2. P.G. Casazza, Approximation properties, In: W.B. Johnson and J. Lindenstrauss (eds.) Handbook of the Geometry of Banach Spaces. Vol. 1, Elsevier, 2001, 271-316.

3. A. Defant and K. Floret, Tensor Norms and Operator Ideals, North-Holland Mathematics Studies, 176, 1993.

4. J. Diestel, Sequences and Series in Banach Spaces, Graduate Texts in Mathematics, 92, Springer-Verlag, New York-Berlin-Heidelbeg-Tokyo, 1984.

5. J. Diestel, H. Jarchow, and A. Tonge, Absolutely Summing Operators, Cambridge studies in advanced mathematics, 43, Cambridge University Press, 1995.

6. J. Diestel and J.J. Uhl Jr., Vector Measures, Mathematical Surveys, 15, Amer. Math. Soc., 1977.

7. H. Fakhoury, Sélections linéaires associées au théorème de Hahn-Banach, J. Funct. Anal. 11 (1972), 436-452.

8. P. Harmand, D. Werner, and W. Werner, M-ideals in Banach Spaces and Banach Algebras, Lecture Notes in Math., 1547, Springer-Verlag, Berlin-Heidelberg, 1993.

9. S. Heinrich and P. Mankiewicz, Applications of ultrapowers to the uniform and Lipschitz classification of Banach spaces, Studia Math. 73 (1982), 225-251.

10. H.E. Lacey, The Isometric Theory of Classical Banach Spaces, Die Grundlehren der mathematischen Wissenschaften in Einzeldarstellungen, 208, Springer-Verlag, Berlin-HeidelbergNew York, 1974.

11. A. Lazar and J. Lindenstrauss, On Banach spaces whose duals are $L_{1}$ spaces, Israel J. Math. 4 (1966), 205-207.

12. A. Lazar and J. Lindenstrauss, Banach spaces whose duals are $L_{1}$ spaces and their representing matrices, Acta Math. 126 (1971), 165-193.

13. A. Lima, V. Lima, and E. Oja, Absolutely summing operators on $C[0,1]$ as a tree space and the bounded approximation property, J. Funct. Anal. 259 (2010), 2886-2901.

14. A. Lima, V. Lima, and E. Oja, Bounded approximation properties via integral and nuclear operators, Proc. Amer. Math. Soc. 138 (2010), 287-297.

15. Å. Lima, V. Lima, and E. Oja, Bounded approximation properties in terms of $C[0,1]$, Math. Scand. 110 (2012), 45-58.

16. Å. Lima and E. Oja, The weak metric approximation property, Math. Ann. 333 (2005), 471-484.

17. V. Lima, The weak metric approximation property and ideals of operators, J. Math. Anal. Appl. 334 (2007), 593-603.

18. J. Lindenstrauss, Extension of compact operators, Memoirs, 48, Amer. Math. Soc., 1964.

19. J. Lindenstrauss and L. Tzafriri, Classical Banach Spaces, Lecture Notes in Math., 338, Springer-Verlag, Berlin - Heidelberg - New York, 1973. 
20. J. Lindenstrauss and D. Wulbert, On the classification of the Banach spaces whose duals are $L_{1}$ spaces, J. Funct. Anal. 4 (1969), 332-349.

21. E. Michael and A. Pełczyński, Separable Banach spaces which admit $\ell_{n}^{\infty}$ approximations, Israel J. Math. 4 (1966), 189-198.

22. E. Oja, The impact of the Radon-Nikodým property on the weak bounded approximation property, Rev. R. Acad. Cien. Serie A. Mat. 100 (2006), 325-331.

23. E. Oja, The strong approximation property, J. Math. Anal. Appl. 338 (2008), 407-415.

24. E. Oja, On bounded approximation properties of Banach spaces, Banach Center Publ. 91 (2010), 219-231.

25. E. Oja, Inner and outer inequalities with applications to approximation properties, Trans. Amer. Math. Soc. 363 (2011), 5827-5846.

26. E. Oja and M. P oldvere, Principle of local reflexivity revisited, Proc. Amer. Math. Soc. 135 (2007), 1081-1088.

27. A. Pietsch, Operator Ideals, North-Holland Publishing Company, Amsterdam-New YorkOxford, 1980.

28. R.A. Ryan, Introduction to Tensor Products of Banach Spaces, Springer Monographs in Mathematics, Springer-Verlag, London, 2002.

29. B. Sims and D. Yost, Linear Hahn-Banach extension operators, Proc. Edinburgh Math. Soc. 32 (1989), 53-57.

30. C. Stegall, Characterizations of Banach spaces whose duals are $L_{1}$ spaces, Israel J. Math. 11 (1972), 299-308.

31. C. Stegall, The Radon-Nikodym property in conjugate Banach spaces, Trans. Amer. Math. Soc. 206 (1975), 213-223.

32. M. Zippin, On some subspaces of Banach spaces whose duals are $L_{1}$ spaces, Proc. Amer. Math. Soc. 23 (1969), 378-385.

${ }^{1}$ Department of Mathematics, University of Agder, Postboks 422 Lundsiden, N-4604 Kristiansand S, NoRWAY.

E-mail address: Asvald.Lima@uia.no

2 Aalesund University College, Postboks 1517, N-6025 Ålesund, Norway.

E-mail address: Vegard.Lima@gmail.com

${ }^{3}$ Faculty of Mathematics and Computer Science, University of Tartu, J. Liivi

2, Ee-50409 Tartu, Estonia; Estonian Academy of Sciences, Kohtu 6, EE-10130

TALLinn, Estonia.

E-mail address: eve.oja@ut.ee 